\title{
An Investigation of a Pre-service Secondary Mathematics Teacher's Conceptual Understanding of Statistical Content and Analysis
}

\author{
Vivian R. Moody ${ }^{1, *}$, Liesel Gardner ${ }^{2}$ \\ ${ }^{1}$ Department of Mathematics, Western Kentucky University, Bowling Green, United States \\ ${ }^{2}$ Department of Mathematics (Teacher Prep Program), Western Kentucky University, Bowling Green, United States \\ *Corresponding author: vivian.moody@wku.edu
}

Received July 17, 2014; Revised July 25, 2014; Accepted July 30, 2014

\begin{abstract}
As part of the requirements for teaching certification, secondary mathematics pre-service teachers enrolled in a teacher preparation program at an American university in the southeastern United States participate in a capstone mathematics course that focuses on conducting research on some topic of interest. This paper is an investigation of one pre-service teacher's challenge to complete this requirement by designing and implementing a research project that required statistical examination and analysis. Findings show that although the pre-service teacher was mathematically able and possessed a depth and breadth of knowledge in mathematics, she struggled with using appropriate statistical methods to carry out the research project. These findings suggest that mathematics teacher education programs need to be intentional about creating opportunities for pre-service teachers to engage in statistical inquiry.
\end{abstract}

Keywords: mathematics teacher preparation, statistics, multiple regression, pearson product-moment correlation, teacher knowledge

Cite This Article: Vivian R. Moody, and Liesel Gardner, "An Investigation of a Pre-service Secondary Mathematics Teacher's Conceptual Understanding of Statistical Content and Analysis." American Journal of Educational Research, vol. 2, no. 8 (2014): 617-623. doi: 10.12691/education-2-8-10.

\section{Introduction}

Mathematics education reform efforts [13,14] have continuously advocated that all students, including preservice teachers need significant study of statistics to adequately and successfully participate in our everchanging global society. However, much research $[1,2,3,5,6]$ continues to document that secondary mathematics teachers often lack sufficient preparation to develop requisite knowledge of statistical content that is needed to effectively teach school statistics. Further, it is well-documented in mathematics and statistics education literature that many mathematics teacher education programs do not provide pre-service teachers sufficient learning experiences in statistical design, implementation, and analysis.

This paper chronicles one secondary mathematics preservice teacher's challenges in designing and implementing a research project that required conceptual understanding of statistical content. The authors sought to answer the following research question: How can opportunities be created in teacher preparation programs to engage secondary mathematics pre-service teachers in statistical inquiry, including data collection and analysis and statistical inference?

\section{Statistical Thinking and Reasoning}

Several research studies [5-10,12-17] in statistics education, as well as mathematics education, document the need to support prospective teachers' development of statistical reasoning and thinking skills. Some scholars $[5,6]$ have argued that while secondary pre-service and inservice mathematics teachers may be very capable in mathematics, they may lack the skills necessary to think and reason through problem situations that require statistical analysis.

Some research studies [7,8,9,10] have identified significant gaps and misconceptions in many pre-service teachers' understanding of statistical concepts and methods. Further, research [10] has found that although some pre-service teachers seem to have an instrumental understanding of statistical concepts, they lack relational understanding of statistics. In other words, these preservice teachers have difficulty understanding the ways in which statistical concepts and measures are related to each other. Moreover, many pre-service mathematics teachers lack exposure to situations that require them to "deal" with data $[7,8,9]$, suggesting that they have had few experiences in collecting, analyzing, and representing data [10]. 
Many scholars $[7,8,9,10]$ have argued that core statistical ideas cannot be treated as mathematical in nature. Further, while statistics is closely related to mathematics, it should not be treated as a subfield of mathematics $[7,8,9,10]$. Thus, while mathematical thinking focuses on abstract patterns, the core of statistical thinking is context. Unique to statistical inquiry is that data or numbers are dealt with within a particular context. Mathematics students, including pre-service mathematics teachers, tend to miss this unique difference between mathematics and statistics, and as a consequence, develop stark misconceptions with regards to statistical content and analysis.

In light of the many research studies that document preservice teachers' misconceptions of statistical concepts and methods, scholars have suggested that in order to increase pre-service teachers' statistical thinking and reasoning skills, they must actively engage in data. Moreover, pre-service teachers must actively engage in the designing and implementation of statistical investigations. The research reported in this paper shares the results of attempting to create an opportunity for a preservice teacher to actively engage in statistical inquiry.

\section{Method}

In this section we describe the secondary mathematics pre-service teacher's (SMPT) design and implementation of a statistical research project. The first author of this paper served as the director of the pre-service teacher's research project. Further, the SMPT and the second author of this paper are one in the same.

\subsection{Capstone Research Project}

Secondary mathematics pre-service teachers (SMPTs) enrolled in a teacher preparation program at an American university in the southeastern United States participate in a capstone research project (CRP) as part of the requirements for teacher certification. The aims of the capstone project are to engage undergraduate mathematics majors in the process of thinking independently, synthesizing mathematical content, and writing and presenting formal mathematics and/or a mathematical idea, concept, or topic. During this project mathematics majors are required to communicate mathematics orally by giving three colloquium talks to an audience of their peers and to mathematics faculty. Also, they are required to communicate mathematics in writing by cultivating a research paper with essential components similar to those of a master's thesis.

The pre-service teacher in this study, hitherto known as She-Roh, designed a statistical investigation based on the popular video game, Call of Duty: Modern Warfare 3. Call of Duty is described as a first-person shooter video game [11]. Like the game's predecessors, it was designed to give its players a movie-like approach to their gaming experience by incorporating objectives, known as missions that can be achieved by employing particular strategies [11]. There are many game modes available that players can choose to play. For purposes of this research, the authors examined four of these different game modes-Domination, Demolition, Kill Confirmed, and Drop Zone.
The objectives of the Domination game mode are to capture three control points on the player's map and hold as many of those points for as long as possible. In Figure 1, She-Roh is shown defending a control point upon its capture.

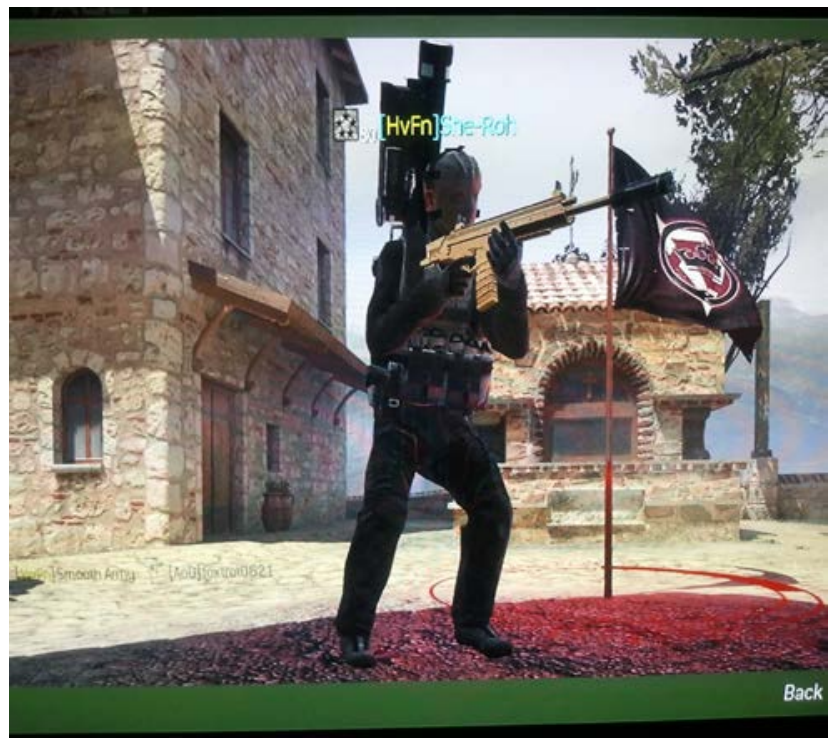

Figure 1. She-Roh Playing Domination Game Mode

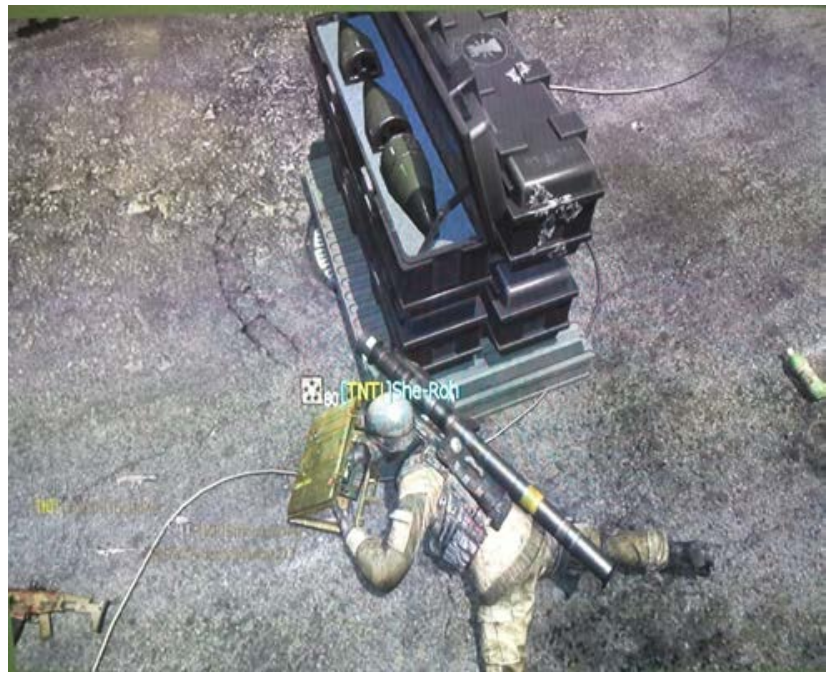

Figure 2. She-Roh Playing Demolition Game Mode

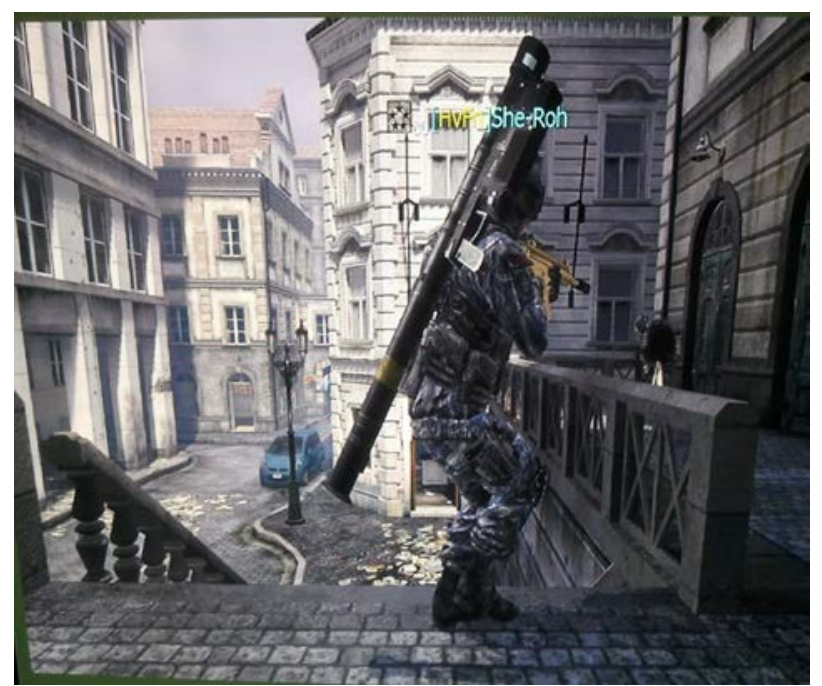

Figure 3. She-Roh Playing Kill Confirmed Game Mode 
In the Demolition game mode the objectives are to attack or defend two bomb sites. If a team of players is attacking, their objectives are to plant bombs and protect them from another team. By contrast, if a team of players is defending, their objective is to diffuse bombs before time expires. Figure 2 shows She-Roh diffusing a bomb while she and her team players were in defense mode.

The objective of the Kill Confirmed game mode is to collect as many dog tags as possible in a given amount of time. Dog tags are miniature metal plates that hang on a chain. Each life-like player in the video game wears a dog tag around his or her neck. When an enemy is eliminated and his dog tag collected, the team is awarded a "kill confirmed.” In Figure 3, She-Roh eliminates an enemy but she or her teammates must collect the enemy's dog tag before the opposing team does. If not, She-Roh's team receives the label "kill denied" and is awarded no points.

In the Drop Zone game mode, players are dropped into a designated area, known as the "red zone.” The objective is to remain alive for as long as possible while in the red zone. Figure 4 shows She-Roh in the red zone as she attempts to remain alive before a particular time interval ends.

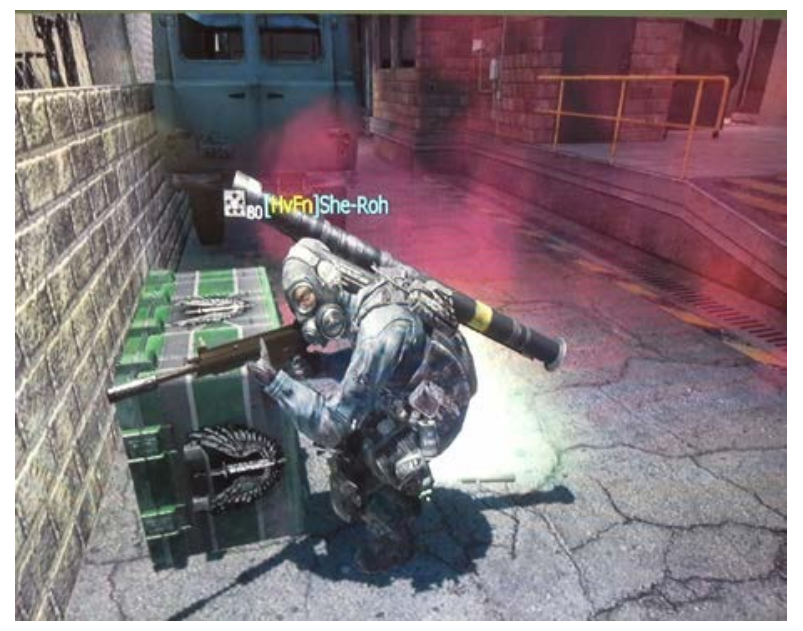

Figure 4. She-Roh Playing Drop Zone Game Mode

\subsection{Data Collection and Analysis}

She-Roh played forty Call of Duty video games and recorded the end-of-game statistics displayed on what is known as a Leader Board. See Figure 5.

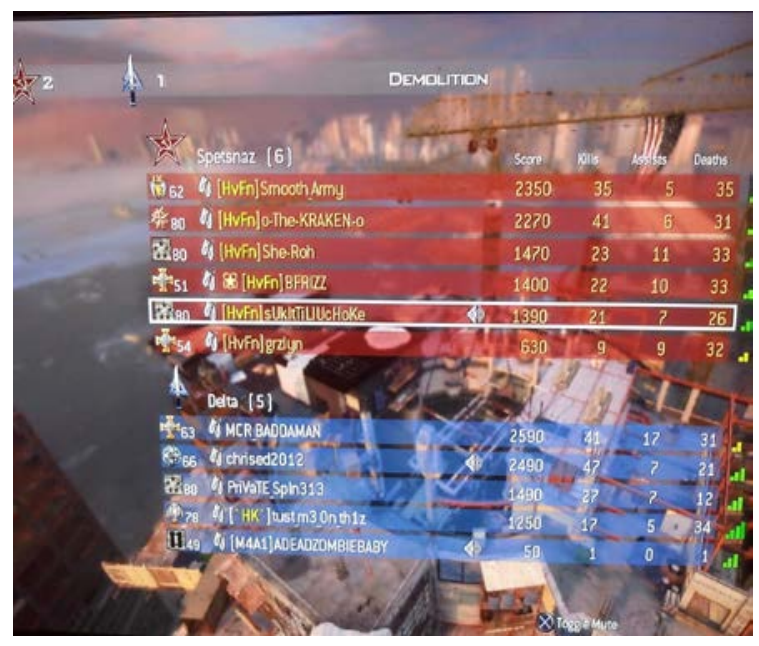

Figure 5. Leader Board in the Demolition Game Mode
Since many variables can affect video game play, including the players' emotional and physical states during game play, the authors identified four variables that were evident in all four game modes. These four variables were each player's number of kills, number of deaths, number of assisted kills, and each player's kills-to-deaths ratio. Note that the number of kills differ from the number of deaths in that kills represent the player's eliminating of an enemy, and the number of deaths represent a player being eliminated by an enemy.

At the end of each game played, She-Roh used the game's built in tracking system of game-play statistics and recorded from the Leader Board the three variables (kills, assists, deaths) for all players, along with all players' overall game score. Although the kills-to-deaths (K/D) ratio was not provided on the Leader Board, She-Roh noted that from her experience of playing the game, she found that most players calculated their K/D ratios and tried to keep this ratio greater than or equal to one. Since the $K / D$ ratio was easy to calculate from the Leader Board's end-of-game statistics, by dividing the number of kills by the number of deaths, She-Roh and her director decided to include the $\mathrm{K} / \mathrm{D}$ ratio as one of the variables that She-Roh would investigate.

The goals of She-Roh's investigation were to analyze end-of-game statistics from the Leader Board for each of the four different game modes and use statistical analyses to make inferences about the variables affecting players' overall game score. She-Roh sought to answer the following questions: (1) To what extent do particular variables, namely kills, assisted kills, deaths, and the kills to deaths ratio, affect a player's overall game score? (2) Is there a significant difference between specific variables given a particular game mode? For example, is there a stronger correlation between kills and overall game score in the Demolition game mode than in the Drop Zone game mode? Further, She-Roh planned to use her analysis to develop a set of scenarios for each game mode that would give a player a competitive edge in achieving the highest score among multiplayers.

She-Roh and the director of She-Roh's CRP (i.e., the authors of this paper) met each week to discuss She-Roh's investigation. Generally during these meetings, She-Roh reported orally and in writing her challenges and progress of carrying out her research project. Specifically, she shared with her director data she collected, her analysis of the data, and to what extent her data collection and analysis answered her research questions.

She-Roh did a preliminary analysis without using a particular statistical method and generally examined the end-of-game statistics for each of the game modes and sought a particular pattern. Confident that equations can be generated from patterns and vice versa, She-Roh decided to examine only her end-of-game statistics that were recorded for each of the ten games played in each game mode and to compare her score with the highest score.

\section{Results of Capstone Research Project}

In this section results from the CRP are presented and discussed. Further, the authors present She-Roh's sense 
making of the data, and her subsequent understanding and communication of the results.

\subsection{Sense Making}

As noted above, She-Roh played a total of forty Call of Duty video games and recorded her end-of-game statistics, as well as the statistics of all players of the game. These forty games were equally divided among four particular game modes - Domination, Demolition, Kill Confirmed, and Drop Zone. In other words, She-Roh played ten Domination games, ten Demolition games, ten Kill Confirmed games, and ten Drop Zone games.

During She-Roh's search for a particular pattern, it appeared to her that there existed a linear relationship between a specific score and a player's overall game score. She-Roh found that as a specific variable (e.g., kills) increases, then a player's overall score increases. To SheRoh, this meant that a linear relationship existed and that there was a positive correlation between the variables (kills, assisted kills, deaths, and kills-to-deaths ratio) being examined and a player's overall game score.

However, She-Roh did not conduct a statistical analysis of the data to determine if a correlational relationship existed between these variables and a player's overall game score. Rather, drawing on her theory that patterns can generate equations and equations can generate patterns, She-Roh attempted to write an equation for each of the four game modes using what she had learned about calculating a player's score while playing the game rather than from the data she had collected from the end-of-game statistics. Table 1 shows She-Roh's equations, which are actually inequalities, for each of the four game modes.

Table 1. Equations She-Roh Generated for Each Game Mode

\begin{tabular}{|c|c|}
\hline $\begin{array}{l}\text { Game } \\
\text { Mode }\end{array}$ & Equation (Inequality) and Corresponding Variables \\
\hline Domination & $\begin{array}{c}\text { SR+[50K+20A+150C+50DC+50H] }>\mathbf{P 1} \\
\text { SR = She-Roh's Score; K = kills; } \mathrm{A}=\text { assists; } \mathrm{C}= \\
\text { captured control points; DC = defended control points; } \\
\mathrm{H} \text { = control points held; } \mathrm{P} 1 \text { = player with highest overall } \\
\text { score }\end{array}$ \\
\hline Demolition & $\begin{array}{c}\text { SR+[50K+20A+100P+100D] }>\mathbf{P 1} \\
\text { SR = She-Roh's Score; K = kills; A = assists; P = planted } \\
\text { bombs; D = diffused bombs; P1 = player with highest } \\
\text { overall score }\end{array}$ \\
\hline $\begin{array}{c}\text { Kill } \\
\text { Confirmed }\end{array}$ & $\begin{array}{c}\text { SR+[50K+50CT+50DT+20A+250T] }>\text { P1 } \\
\text { SR = She-Roh's Score; K = kills; } \mathrm{A}=\text { assists; } \mathrm{CT}= \\
\text { collected tags; DT = denied tags; } \mathrm{T}=\text { collecting own tags; } \\
\text { P1 = player with highest overall score }\end{array}$ \\
\hline Drop Zone & $\begin{array}{c}\text { SR+[50K+20TS+20A+25SP] }>\mathbf{P 1} \\
\text { SR = She-Roh's Score; } \mathrm{K}=\text { kills; } \mathrm{A}=\text { assists; } \mathrm{TS}=\text { time } \\
\text { in seconds; } \mathrm{SP}=\text { share packages; } \mathrm{P} 1 \text { = player with highest } \\
\text { overall score }\end{array}$ \\
\hline
\end{tabular}

She-Roh then attempted to enter her equations (i.e., inequalities) into a matrix and use Mathematica to find a solution. As detailed below, She-Roh was very disappointed to find that there was no solution to the inequalities she had generated.

\subsection{A Second Look}

She-Roh wrote in her research paper, "After many attempts to solve the matrix and [being unsuccessful], a new approach was necessary." She-Roh explained further: Given the many uncontrollable variables in playing video games, it is difficult to isolate specific methods or mathematical equations in a small amount of time to be a better player. However, just being able to understand where the points come from and how to maximize them makes a big difference in competitive game play. Recall, one of She-Roh's goals was to use her analysis and the results to develop a set of scenarios for each game mode that would give a player a competitive edge in achieving the highest score among multiplayers.

Given that She-Roh could not find a solution to her system of linear equations (inequalities) she generated from "playing" each of the game modes, She-Roh and her director decided to take a second, more thorough look at the data collected (i.e., end-of-game statistics) from the Leader Board. At this juncture, She-Roh was challenged to analyze these data by choosing an appropriate statistical method that would answer her research questions: (1) To what extent do particular variables, namely kills, assisted kills, deaths, and kills-to-deaths ratios, affect a player's overall game score? (2) Is there a significant difference between specific variables given a particular game mode? Unable to determine a statistical method that would help her answer her research questions, She-Roh continued to seek an equation or system of equations.

In evaluating her score compared to other players, SheRoh concluded that it would be beneficial to investigate the difference between her score and the highest score for each game played and for each of the game modes. Hence, She-Roh calculated the deficit average of her score and the highest score for each game she played in each game mode. She plotted the results and concluded that she was more successful at the Drop Zone game mode. The graph She-Roh generated is shown in Figure 6.

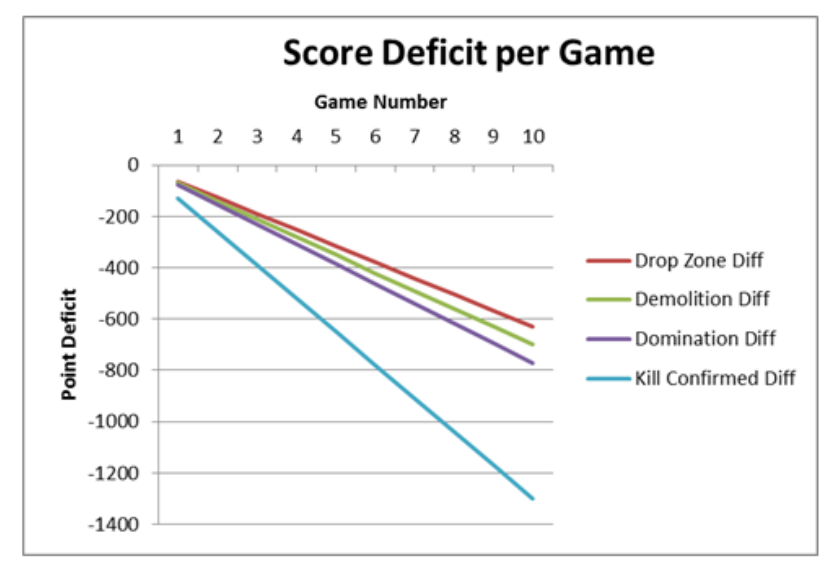

Figure 6. She-Roh's Score Deficit Graph

She-Roh examined the data further, calculating averages for each of the variables (scores, kills, deaths, assists, K/D ratio) and again sought a pattern. She concluded that in the Drop Zone game mode, it seemed as though the variable "kills" did not significantly affect a player's overall game score as it had in the other game modes. She-Roh also noted that in examining her number of "kills" per game, it was apparent that her number was significantly lower than that of other players. Using this discovery and the graph (Figure 6) she plotted, she concluded that the slope determined in the Drop Zone was closest to a positive slope than any of the other game modes. She now believed that other players could use her method to determine which of the game modes would yield the best score for that player. 
Although She-Roh was quite pleased with this discovery, she was still dismayed that she could not find a solution to the system of equations (inequalities) she generated earlier. (See Table 1) She-Roh wrote the following in the conclusion of her research paper: Given the time constraints of this [capstone] research project, it is not possible to cover every possible outcome or strategy to make a solid conclusion. However, it would be something worth studying further.

\section{Discussion}

For purposes of this paper, the first author investigated She-Roh's complex endeavors to complete her capstone research project. The discussion that follows is the first author's synthesis of She-Roh's challenges in understanding statistical content and methods. This discussion is also the first author's attempt to fit SheRoh's efforts within the broader context of teacher education.

\subsection{Correlation: One Missed Opportunity}

Recall, She-Roh discovered that there existed some type of linear relationship between particular variables and a player's overall game score. However, she had difficulty using the data she collected to investigate this relationship. With more time to complete her project and perhaps with more guidance from her director, it is quite probable that She-Roh would have pieced together this notion of a linear relationship between variables, their apparent correlations, and regression analysis.

First, it would have been useful to seek whether a linear correlation existed between the variables that were identified in each of the game modes. Further, the use of a mathematical measurement to describe the strength of this linear relationship would have been appropriate. Note that an appropriate measure to use in this case is the Pearson product-moment correlation coefficient, "named in honor of the English statistician Karl Pearson (1857-1936), who is credited with formulating [the coefficient]" [4].

One missed opportunity in She-Roh's research project was to use her sample data and determine this correlation coefficient for each of the game modes. Shown in Table 2, we see that in the Domination game mode, there is a strong, positive relationship between a player's overall game score and the player's number of kills. This means that if we consider only a player's overall game score and the number of kills the player achieves during a game, and if we do not consider any of the other variables, then about $77 \%$ of the variation in a player's overall game score can be explained by the corresponding variation in the player's number of kills. In examining whether the explanatory variables are closely related to each other, we also find in Table 2 that the explanatory variable, "kills" is positively related to the explanatory variable, "K/D ratio."

Table 2. Domination Game Mode Correlation Coefficients

\begin{tabular}{|c|c|c|c|c|c|}
\hline & Score & Kills & Assists & Deaths & K/D Ratio \\
\hline Score & 1 & & & & \\
\hline Kills & $\mathbf{0 . 7 6 7 8}$ & 1 & & & \\
\hline Assists & 0.1504 & 0.4209 & 1 & & \\
\hline Deaths & 0.0634 & 0.0682 & 0.2927 & 1 & \\
\hline K/D Ratio & 0.5572 & $\mathbf{0 . 7 2 6 8}$ & 0.0828 & -0.5460 & 1 \\
\hline
\end{tabular}

With regards to the Demolition game mode, as shown in Table 3, there is a very strong, positive correlation between a player's overall game score and the number of kills the player achieves during game play. Thus, for this particular game mode, about $98 \%$ of the variation in a player's overall game score can be explained by the corresponding variation in the number of kills a player achieves during game play. Interestingly, we find no other "strong" relationships between any of the other variables.

Table 3. Demolition Game Mode Correlation Coefficients

\begin{tabular}{|c|c|c|c|c|c|}
\hline & Score & Kills & Assists & Deaths & K/D Ratio \\
\hline Score & 1 & & & & \\
\hline Kills & $\mathbf{0 . 9 7 6 0}$ & 1 & & & \\
\hline Assists & 0.4010 & 0.3223 & 1 & & \\
\hline Deaths & 0.4549 & 0.3947 & 0.6236 & 1 & \\
\hline K/D Ratio & 0.3912 & 0.4562 & -0.1893 & -0.4697 & 1 \\
\hline
\end{tabular}

In the Kill Confirmed game mode, we again find a strong correlation between a player's overall game score and the number of kills achieved. See Table 4. Also, as was the case with Domination game mode, we find that the variable "kills" is closely related to the variable "K/D ratio.”

Table 4. Kill Confirmed Game Mode Correlation Coefficients

\begin{tabular}{|c|c|c|c|c|c|}
\hline & Score & Kills & Assists & Deaths & K/D Ratio \\
\hline Score & 1 & & & & \\
\hline Kills & $\mathbf{0 . 8 2 7 3}$ & 1 & & & \\
\hline Assists & 0.2617 & 0.0304 & 1 & & \\
\hline Deaths & 0.3896 & 0.0813 & 0.2519 & 1 & \\
\hline K/D Ratio & 0.5170 & $\mathbf{0 . 7 8 4 7}$ & -0.1487 & -0.4565 & 1 \\
\hline
\end{tabular}

As shown in Table 5, in the Drop Zone game mode, we find no "strong" correlations between a player's overall game score and any of the explanatory variables. Recall that She-Roh found this to be the case by calculating her average game score and her number of kills and comparing these with the averages of the player with the highest score. Also shown in Table 5, we find that the variable "kills" is related to the variable "K/D ratio." This is also the case with "deaths" and the "K/D ratio." What is interesting is that according to our Pearson correlation, there is virtually no relationship between deaths and a player's overall game score in the Drop Zone game mode. In conversations with her director, She-Roh stated that she was better at the Drop Zone game mode because she only needed to stay alive while in the zone and not necessary eliminate any enemies. However, as shown in Table 5, it seems that other variables would better explain the variation in a player's overall game score.

Table 5. Drop Zone Game Mode Correlation Coefficients

\begin{tabular}{|c|c|c|c|c|c|}
\hline & Score & Kills & Assists & Deaths & K/D Ratio \\
\hline Score & 1 & & & & \\
\hline Kills & $\mathbf{0 . 4 8 2 5}$ & 1 & & & \\
\hline Assists & 0.2169 & 0.2601 & 1 & & \\
\hline Deaths & -0.0085 & -0.0486 & 0.1556 & 1 & \\
\hline K/D Ratio & 0.3200 & $\mathbf{0 . 7 2 5 5}$ & 0.0726 & $\mathbf{- 0 . 6 1 0 0}$ & 1 \\
\hline
\end{tabular}

\subsection{Multiple Regression: A Second Missed Opportunity}

She-Roh's attempt to develop a system of equations and find a solution to this system would have been less difficult with the use of appropriate statistical terminology and notation. In essence, She-Roh sought to predict or forecast a player's overall game score base on a particular 
set of variables. Using statistics, we can describe the mathematical formula for expressing linear relationships among more than two variables as follows:

$$
y=b_{0}+b_{1} x_{1}+b_{2} x_{2}+b_{3} x_{3}+\ldots+b_{k} x_{k}
$$

In Equation (1), $y$ is the variable we want to predict or forecast, namely, our response variable. The $k$ variables $x_{1}, x_{2}, x_{3}, \ldots, x_{k}$ are the specified variables on which $\mathrm{y}$ is going to be based and are known as our explanatory variables. In terms of She-Roh's project, $y$ would have been a player's overall game score, and the $k$ variables would have been the number of kills, deaths, and assists a player achieved during a particular game played, and a final $k$ variable would have been the player's K/D ratio. In this sense, the $k$ variables "explain" the response variable $y$. In Equation (1), $b_{0}, b_{1}, b_{2}, \ldots, b_{k}$ are numerical constants or coefficients. The numerical values of these coefficients must be mathematically determined from given data and are obtained from what is statistically known as the leastsquares criterion [4]. "In multiple regression, the leastsquares criterion states that the following sum (over all data points),

$$
\sum\left[y_{i}-\left(b_{0}+b_{1} x_{1 i}+b_{2} x_{2 i}+b_{3} x_{3 i}+\ldots+b_{k} x_{k i}\right)\right]^{2}
$$

must be made as small as possible” [4]. In Equation (2), $y_{\mathrm{i}}$ is the $i$ th data value for $y ; x_{1 i}$ is the ith data value for $x_{1} ; x_{2 i}$ is the ith value for $x_{2}$; and $x_{k i}$ is the ith data value for $x_{k}$. Therefore, since Equation (1) gives the predicted value of $y$, we have that the equation

$$
y_{i}-\left(b_{0}+b_{1} x_{1 i}+b_{2} x_{2 i}+b_{3} x_{3 i}+\ldots+b_{k} x_{k i}\right)
$$

represents the difference between the observed $y$ value and the predicted $y$ value. Thus, "when we square this difference and total the result over all data points and choose the values of $b_{0}, b_{1}, b_{2}, \ldots, b_{k}$ to minimize the sum [i.e., minimize Equation (2)], then we are satisfying the least-squares criterion" [4]. Further, in the multiple regression model, the least-squares criterion can be reduced to solving a system of linear equations. Thus, She-Roh's idea to generate a system of equations and find a solution was very much appropriate. However, she generated her equations (inequalities) based on her playing the game and what she observed during game play to calculate a player's score rather than generating the equations based on the data she collected with a particular set of variables.

Suppose She-Roh had determined that two explanatory variables would adequately "explain" the response variable $y$, or a player's overall game score. The system of equations to solve is as follows:

$$
\begin{aligned}
\sum y_{i} & =n b_{0}+b_{1}\left(\sum x_{1 i}\right)+b_{2}\left(\sum x_{2 i}\right) \\
\sum x_{1 i} y_{i} & =b_{0}\left(\sum x_{1 i}\right)+b_{1}\left(\sum x_{1 i}{ }^{2}\right)+b_{2}\left(\sum x_{1 i} x_{2 i}\right) \\
\sum x_{2 i} y_{i} & =b_{0}\left(\sum x_{2 i}\right)+b_{1}\left(\sum x_{1 i} x_{2 i}\right)+b_{2}\left(\sum x_{2 i}{ }^{2}\right)
\end{aligned}
$$

In this system (4), $\mathrm{n}$ represents the number of data points, and the $\mathrm{x}$ and $\mathrm{y}$ variables represent given data values. The only unknowns are $b_{0}, b_{1}$, and $b_{2}$. Thus, we use the system (4) to solve for these unknowns. As one can see, solving this system (4) is quite complicated and even more so in the case of more than two explanatory variables as in She-Roh's research project. Thus, with regards to She-Roh's research project, it would have been more beneficial for her to use a statistical software package to perform a multiple regression analysis on the data she collected for each of the game modes.

A multiple regression analysis of the data She-Roh collected for the Domination game mode was performed using Excel. The results include the coefficients $b_{0}, b_{1}$, and $b_{2}$, standard errors for each of the explanatory variables, t-Stat values, and the Lower and Upper 95\% confidence intervals for the coefficients. For purposes of this paper, we focus only on the R-square value and then the P-values for each of the explanatory variables. These are shown in Table 6.

Table 6. Domination Game Mode

\begin{tabular}{|c|c|c|c|c|}
\hline \multicolumn{2}{|c|}{ Regression Statistics } & & $\mathrm{t}$ - Stat & P- value \\
\hline Multiple R & 0.7946 & Intercept & 1.6318 & 0.1102 \\
\hline R Square & 0.6314 & Kills & 3.7279 & 0.0005 \\
\hline Adjusted R Square & 0.5963 & Assists & -2.1745 & 0.0354 \\
\hline Standard Error & 347.0094 & Deaths & 0.1243 & 0.9017 \\
\hline Observations & 47 & K/D Ratio & -0.3034 & 0.7630 \\
\hline
\end{tabular}

The multiple regression analysis yielded an R-Square value of 0.6314 . This tells us that about $63 \%$ of the variation in the response variable (i.e., a player's overall game score) can be explained from the least-squares regression equation (1) and the corresponding joint variation of the explanatory variables (i.e., kills, assists, deaths, K/D ratio) taken together. This R-Square value also means that the remaining $37 \%$ of the variation in a player's overall game score is due to random chance or other variables that were not included in this regression equation.

The P-value results shown in Table 6 provide further information about the explanatory variables in the Domination game mode and their level of significance. Pvalue results show that since the P-values for kills and assists are less than the level of significance $\alpha=0.05$, we can conclude that at the $5 \%$ level of significance that these variables (kills and assists) should be included as explanatory variables in the least-squares equation.

As shown in Table 7, our R-square value of 0.9617 means that about $96 \%$ of the variation in the response variable (i.e., a player's overall game score) can be explained from the least-squares equation and the corresponding joint variation of the explanatory variables taken together. Also, given the P-value results, we can conclude that at the $5 \%$ level of significance that the variable "kills" should be included as an explanatory variable in the least-squares equation, whereas the other variables have less influence on a player's overall game score.

Table 7. Demolition Game Mode

\begin{tabular}{|c|c|c|c|c|}
\hline \multicolumn{2}{|c|}{ Regression Statistics } & & $\mathrm{t}$ - Stat & P- value \\
\hline Multiple R & 0.9807 & Intercept & 0.6022 & 0.5504 \\
\hline R Square & 0.9617 & Kills & 17.172 & $4.65 \mathrm{E}-20$ \\
\hline Adjusted R Square & 0.9579 & Assists & 1.9004 & 0.0646 \\
\hline Standard Error & 116.2549 & Deaths & 0.4653 & 0.6444 \\
\hline Observations & 45 & K/D Ratio & -0.1970 & 0.8449 \\
\hline
\end{tabular}

As shown in Table 8, we find quite interesting results with regards to the Kill Confirmed game mode. First, 83\% of the variation in a player's overall game score can be explained from the least-squares equation and the corresponding explanatory variables taken together. 
Second, the P-value results show that all four explanatory variables are significant at the $5 \%$ confidence level, which means that all four variables should be included as an explanatory variable in the least-squares equation for this particular game mode.

Table 8. Kill Confirmed Game Mode

\begin{tabular}{|c|c|c|c|c|}
\hline \multicolumn{2}{|c|}{ Regression Statistics } & & $\mathrm{t}-$ Stat & P-value \\
\hline Multiple R & 0.9123 & Intercept & -1.8174 & 0.0763 \\
\hline R Square & 0.8322 & Kills & 2.7649 & 0.0084 \\
\hline Adjusted R Square & 0.8162 & Assists & 2.7928 & 0.0078 \\
\hline Standard Error & 345.8051 & Deaths & 4.1335 & 0.0002 \\
\hline Observations & 47 & K/D Ratio & 2.083 & 0.0434 \\
\hline
\end{tabular}

Consistent with She-Roh's findings, in Table 9, we see that all of the explanatory variables can be "excluded" from the least-squares, as each has little or no influence on a player's overall game score. Specifically, only $24 \%$ of the variation in a player's overall game score can be explained from the least-squares equation and the corresponding joint variation of the explanatory variables taken together.

Table 9. Drop Zone Game Mode

\begin{tabular}{|c|c|c|c|c|}
\hline \multicolumn{2}{|c|}{ Regression Statistics } & & $\mathrm{t}$ - Stat & P-value \\
\hline Multiple R & 0.4944 & Intercept & 3.2937 & 0.0020 \\
\hline R Square & 0.2444 & Kills & 1.9773 & 0.0643 \\
\hline Adjusted R Square & 0.1756 & Assists & 0.6910 & 0.4933 \\
\hline Standard Error & 571.1169 & Deaths & -0.3351 & 0.7391 \\
\hline Observations & 49 & K/D Ratio & -0.3953 & 0.6946 \\
\hline
\end{tabular}

\section{Implications and Conclusions}

In examining She-Roh's efforts in statistical inquiry, we find that She-Roh's knowledge and understanding of statistical methods existed but in boxes or compartments that did not relate one to the other. In other words, SheRoh began her project identifying a response or dependent variable and several explanatory or independent variables. However, She-Roh had difficulty making this connection. Further, She-Roh made an appropriate assumption that she could predict or forecast an overall game score for each of the four game modes based on data collected from each of these. However, She-Roh's efforts were void of implementing any type of statistical method to analyze the data collected. She relied heavily on what she had learned from playing the game and from her observations of how the gaming system calculated scores during game play.

In comparing the statistical methods that could have been applied to She-Roh's investigation and the methods she ultimately used, it is apparent that She-Roh would have benefitted from more explicit guidance to help her make the connections that exist among statistical concepts, measures, and representations of data. Although we cannot generalize She-Roh's case to explain the difficulties that some pre-service mathematics teachers encounter when required to use statistics, She-Roh's case does provide yet another example of why mathematics teacher education programs must provide more salient opportunities for pre-service teachers to engage in statistical inquiry.

\section{References}

[1] Arnold, P, "Developing new statistical content knowledge with secondary school mathematics teachers," in Proceedings of the ICMI Study 18 and 2008 IASE Round Table Conference, Springer.

[2] Batanero, C., Burrill, G. and Reading, C (Eds.), Teaching statistics in school mathematics_challenges for teaching and teacher education. Springer, Dordrecht, 2011.

[3] Ben-Zvi, D. and Garfield, J, The challenge of developing statistical literacy, reasoning, and thinking, Kluwer, Dordrecht, 2004.

[4] Brase, C. H. and Brase, C. P, Understanding statistics: concepts and methods, Houghton Mifflin Company, New York, 2008.

[5] Cobb, G. W. and Moore, D. S, "Mathematics, statistics, and teaching," The American mathematical monthly, 104 (9), 801-823, 1997.

[6] delMas, R., Garfield J., Ooms, A. and Chance, B, “Assessing students' conceptual understanding after a first course in statistics," Statistics education research journal, 6 (2), 28-58, 2007.

[7] Levy, A. M, "Indexing distributions of data: pre-service teachers' notion of representativeness," School science and mathematics 104 (3), 119-134, 2004.

[8] Levy, A. M, "Using data comparison to support a focus on distribution: examining pre-service teachers' understandings of distribution when engaged in statistical inquiry," Statistics education research journal, 5 (2), 89-114, 2006.

[9] Leavy, A. M. and O’Loughlin, N, "Moving beyond the arithmetic average: pre-service teachers' understanding of the mean," Statistics education research journal, 9 (1), 53-90, 2006.

[10] Leavy, A. M. and Sloane, F, Developing statistical literacy in primary level mathematics, Stationery, Dublin, 2010.

[11] Mann, K, Delta gamer: the history of The Call of Duty game engine mechanics, Activision, 2011.

[12] Moore, D.S, "New pedagogy and new content: the case of statistics,” Statistics education research journal, 65, 123-137, 1997.

[13] National Council of Teachers' Mathematics, Principles and standards for school mathematics, NCTM, Reston, 2000.

[14] National Council of Teachers' Mathematics, Professional Teaching Standards for School Mathematics, NCTM, Reston, 1992.

[15] Peters, S. A, “A robust understanding of variation,” Statistics education research journal, 10 (1), 52-88, 2011.

[16] Shaughnessy, J. M, "Research in probability and statistics: reflections and directions," in Handbook of research on mathematical teaching and learning, McMillan, New York, 1992, 465-494.

[17] Zientek, L. R., Carter, T. A., Taylor, J. M. and Capraro, R. M, "Preparing prospective teachers: an examination of attitudes toward statistics," Journal of mathematical sciences and mathematics education, 5 (1), 25-38, 2011. 